\title{
Two-to-three dimensional transition in neutral gold clusters: The crucial role of van der Waals interactions and temperature
}

\author{
Bryan R. Goldsmith, ${ }^{1,2, *}$ Jacob Florian, ${ }^{2}$ Jin-Xun Liu, ${ }^{2}$ Philipp Gruene, ${ }^{1}$ Jonathan T. Lyon, ${ }^{1,3}$ David M. Rayner, ${ }^{4}$ \\ André Fielicke, ${ }^{1, \dagger}$ Matthias Scheffler, ${ }^{1}$ and Luca M. Ghiringhelli ${ }^{1, \ddagger}$ \\ ${ }^{1}$ Fritz-Haber-Institut der Max-Planck-Gesellschaft, Faradayweg 4-6, D-14195 Berlin, Germany \\ ${ }^{2}$ Department of Chemical Engineering, University of Michigan, Ann Arbor, Michigan 48109-2136, USA \\ ${ }^{3}$ Department of Chemistry and Biochemistry, Kennesaw State University, 370 Paulding Avenue Northwest, MD 1203, \\ Kennesaw, Georgia 30144, USA \\ ${ }^{4}$ National Research Council, 100 Sussex Drive, Ottawa, Ontario, Canada K1A OR6
}

(Received 19 November 2018; revised manuscript received 23 December 2018; published 18 January 2019)

\begin{abstract}
We predict the structures of neutral gas phase gold clusters $\left(\mathrm{Au}_{n}, n=5-13\right)$ at finite temperatures based on free-energy calculations obtained by replica-exchange $a b$ initio molecular dynamics. The structures of neutral $\mathrm{Au}_{5}-\mathrm{Au}_{13}$ clusters are assigned at $100 \mathrm{~K}$ based on a comparison of experimental far-infrared multiple photon dissociation spectra performed on Kr-tagged gold clusters with theoretical anharmonic IR spectra and free-energy calculations. The critical gold cluster size at which the most stable isomer changes from planar to nonplanar is $\mathrm{Au}_{11}$ (capped-trigonal prism, $\mathrm{D}_{3 h}$ ) at $100 \mathrm{~K}$. However, at $300 \mathrm{~K}$ (i.e., room temperature), planar and nonplanar isomers may coexist even for $\mathrm{Au}_{8}, \mathrm{Au}_{9}$, and $\mathrm{Au}_{10}$ clusters. Density-functional theory exchange-correlation functionals within the generalized gradient or hybrid approximation must be corrected for long-range van der Waals interactions to accurately predict relative gold cluster isomer stabilities. Our paper gives insight into the stable structures of gas phase gold clusters by highlighting the impact of temperature, and therefore the importance of free energy over total-energy studies, and long-range van der Waals interactions on gold cluster stability.
\end{abstract}

DOI: 10.1103/PhysRevMaterials.3.016002

\section{INTRODUCTION}

Metal clusters in the gas phase are widely used as model systems to study fundamental properties of condensed matter (see, e.g., [1-7]). At the nanoscale, gold is not a fully noble, nonreactive material; thus, gold clusters are of particular interest due to their possible applications in gas sensing, pollution reduction, and catalysis [8-17]. Gold clusters in the gas phase exhibit many structural isomers of similar energetics and can rapidly interconvert among them [18-24]. Geometry and size can impact the physicochemical properties of clusters, for example, the gap between the highest occupied molecular orbital and the lowest unoccupied molecular orbital, polarizability, and catalytic activity [25-31]. Small gold clusters often adopt stable planar geometries [32-36]. The critical size at which gold clusters begin favoring nonplanar (threedimensional, 3D) structures over planar (two-dimensional, 2D) structures has attracted sustained interest [37-44]. Gas phase ion-mobility experiments at room temperature suggest a transition from a $2 \mathrm{D}$ to $3 \mathrm{D}$ ground-state structure at size 12 [45] and 8 [46] for negatively and positively charged clusters, respectively. Computational and experimental evidence supports these ground-state structural assignments for the 2D to 3D transition size $[47,48]$. For neutral gold clusters,

\footnotetext{
*bgoldsm@umich.edu

${ }^{\dagger}$ fielicke@fhi-berlin.mpg.de

${ }^{\ddagger}$ ghiringhelli@fhi-berlin.mpg.de
}

computational studies predict their critical transition size is between $\mathrm{Au}_{10}$ and $\mathrm{Au}_{14}$ at $0 \mathrm{~K}[26,38,44]$, but experimental evidence is lacking due to the difficulty in spectroscopically characterizing neutral gas phase clusters relative to charged clusters.

Both experimental and computational studies indicate that dynamic structural rearrangements are a common feature among clusters at finite temperatures [22,49-57]. It is clear that small gold clusters favor a 2D structure. The critical size at which gold clusters favor $3 \mathrm{D}$ structures over 2D structures likely depends on temperature, but few studies have computationally examined the isomer stability at finite temperature $[49,57,58]$. One study used metadynamics to probe the free-energy surface of neutral $\mathrm{Au}_{12}$ and predicted dynamic coexistence of multiple planar and nonplanar isomers at room temperature [58].

Besides temperature effects on cluster stability, the longrange tail contributions of van der Waals (vdW) interactions in Kohn-Sham density-functional theory (DFT) studies of clusters have been neglected in most previous works. Van der Waals interactions can be crucial for predicting the stability of materials and molecules [59-63], molecule adsorption to surfaces [64], and even selectivity of reactions [65]. Including $\mathrm{vdW}$ interactions in DFT calculations has been suggested to stabilize 3D isomers relative to $2 \mathrm{D}$ isomers for small gold clusters [57,66,67]; nonetheless, the magnitude of vdW interactions on gold cluster isomer stability over a broad size range has not been quantified. Ultimately, accurate first-principles predictions, including both temperature effects (i.e., enthalpic 
and entropic contributions to obtain free energies) and $\mathrm{vdW}$ interactions, combined with experimental characterization, are needed to unambiguously identify the structures of clusters at finite temperature.

The structures of $\mathrm{Au}_{3}-\mathrm{Au}_{8}$ at $100 \mathrm{~K}$ have been previously determined by some of the authors via a combination of both first-principles modeling and far-infrared multiple photon dissociation (FIR-MPD) spectroscopy [55,66]. Here, we predict the structures of neutral gas phase gold clusters $\left(\mathrm{Au}_{n}, n=5-13\right)$ at finite temperatures based on free-energy calculations using replica-exchange $a b$ initio molecular dynamics combined with the multistate Bennett acceptance ratio (REMD+MBAR) [68]. We then compare experimental FIRMPD spectra performed on Kr-tagged gold clusters with theoretical anharmonic IR spectra and free-energy calculations to structurally assign $\mathrm{Au}_{9}-\mathrm{Au}_{13}$, where the transition from planar to three-dimensional structures is expected to occur for neutral gold clusters.

\section{METHODS}

\section{A. Computational}

The FHI-aims package is used for all electronic-structure calculations $[69,70]$. FHI-aims gives an accurate all-electron, full-potential description based on numeric atom-centered basis functions. Since the gold cluster ground-state structures are expected to depend on the theoretical method used $[44,48]$, the lowest-energy isomers are examined at various levels of DFT and beyond. The exchange-correlation (xc) densityfunctional approximations (DFAs) that we use are the PerdewBurke-Ernzerhof (PBE) xc functional [71] and the HeydScuseria-Ernzerhof (HSE06) hybrid xc functional [72,73]. Because these semilocal xc functionals do not account for the long-range vdW interactions, here we augment our DFAs with long-range vdW corrections using both the pairwise Tkatchenko-Scheffler (TS) [74] method and the many-body dispersion (MBD) approach with range separated self consistent screening (rsSCS) [75,76]. We call these approximations $\mathrm{DFA}+\mathrm{TS}$ and DFA+MBD, where DFA is replaced by the name of the xc functional used to approximate the energy. For HSE06 calculations we use a screening parameter of $0.11 /$ bohr and $25 \%$ exact exchange [77].

Geometry optimizations used (collinear) spin-polarized DFT with "tight" integration grid settings and accurate "tier 2" basis sets, unless stated otherwise. Clusters are treated in their ground-state electronic configurations-even-sized clusters are singlets, whereas odd-sized configurations are doublets. The fundamental gaps of the gold clusters are nonzero at the studied temperatures [30]. All DFA+TS and DFA+MBD geometry optimization calculations are compared against random phase approximation (RPA) (exact exchange plus electronic correlation in the RPA) single-point calculations, starting from PBE orbitals (denoted as RPA@PBE) [78]. RPA calculations have been used previously to study the stability of gas phase gold clusters [44]. Our RPA@PBE calculations were corrected for basis-set superposition error and used "very-tight" integration grid settings with the large cc-pV5ZPP basis from the EMSL basis-set exchange database [79]. Zero-point energy corrections are not included because they are typically not required for atomic clusters. Gold relativistic effects are treated using the atomic ZORA scalar correction [80]. Spin-orbit coupling is not included in any calculations [37,81]. A prior study suggested spin-orbit coupling only marginally affects the predicted ground-state structure for small gold clusters [37].

We use REMD because it allows for an unbiased search of the potential-energy surface from which gold cluster structures can be identified [82]. REMD simulations used energies and forces obtained from spin-polarized DFT using $\mathrm{PBE}+\mathrm{MBD}$ with "light" integration grid settings and "tier 1 " basis sets. REMD simulations used between 10 and 15 replicas distributed over the temperature range 100-1000 K. Each replica was initialized as a randomly generated structure. Between attempted replica exchanges, constant-Number-ofparticles, constant Volume, constant Temperature (i.e., NVT) Born-Oppenheimer $a b$ initio molecular dynamics ran for 0.5 ps using a 10-fs time step. The simulation time per replica was $300 \mathrm{ps}$, resulting in a combined simulation time of at least $3 \mathrm{~ns}$ for each $\mathrm{Au}_{n}$ cluster $(n=5-13)$. The stochastic velocity rescaling thermostat was used to sample the canonical $(N V T)$ ensemble with a $\tau$ parameter of $100 \mathrm{fs}$ [83]. Cluster configurations and potential energies were subsampled (with lag time based on the decay of the velocity autocorrelation of the simulation trajectories) from the REMD simulations to obtain statistically independent samples to compute free-energy surfaces via the MBAR using the pyMBAR software [68]. MBAR is a direct extension of BAR that allows for assessing data from all REMD states (here, a state is identified by the temperature) to predict free energies. The dimensionless free energies $\left(\beta \Delta F\right.$, where $\left.\beta=1 / k_{B} T\right)$ for $\mathrm{Au}_{5}-\mathrm{Au}_{13}$ have an average error of $1.1 \pm 0.3 \beta \Delta F$. Note, our analysis neglects the small rotational free-energy contribution to the total free energy because the components of the forces yielding rigid-body rotations are eliminated to avoid the inconvenient "ice-cube effect" [84,85], which is otherwise unavoidable in molecular dynamics simulations of an isolated molecule or cluster. All the gold cluster configurations can be downloaded from the NOMAD Repository [86].

Theoretical anharmonic IR spectra of $\mathrm{Au}_{9}-\mathrm{Au}_{13}$ at $100 \mathrm{~K}$ were computed from Born-Oppenheimer $a b$ initio moleculardynamics trajectories in the canonical ensemble (using the stochastic velocity rescaling thermostat) by evaluating the ensemble average of the Fourier transform of the dipoledipole time-autocorrelation function $[55,87,88]$. In this way, anharmonic effects are fully included in the calculations. These simulations were $100-150$ ps in duration and used energies and forces obtained from spin-polarized DFT with $\mathrm{PBE}+\mathrm{MBD}$ and "tight" integration settings and "tier 2" basis sets. Although $\mathrm{Kr}$ is present in the FIR-MPD spectroscopy experiments, we neglect $\mathrm{Kr}$ in our theoretical anharmonic IR spectra because its impact on the gold cluster vibrational spectra is likely negligible at the cluster sizes considered in this paper-the effect of $\mathrm{Kr}$ on the IR spectrum for $\mathrm{Au}_{7}$ was minimal in a prior study by some of the authors [55]. Pendry reliability factors (Pendry $R$ factor, $R_{P}$ ) were computed to give a quantitative comparison of how well theoretical IR spectra peak positions and their intensities agree with experimental FIR-MPD spectra. $R_{P}$ compares peak positions between two spectra using the renormalized logarithmic derivatives of their intensities [89], which results in $R_{P}=0$ for perfectly 
agreeing spectra, $R_{P}=1$ for uncorrelated spectra, and $R_{P}=$ 2 for anticorrelated spectra. Because $R_{P}$ is sensitive to noise and needs well-separated peaks, we smoothed the experimental FIR-MPD spectra four times and theoretical IR spectra twice using a three-point formula with a $0.6-\mathrm{cm}^{-1}$ grid separation [90]. A constant half width at half maximum of $5 \mathrm{~cm}^{-1}$ was used when computing $R_{P}$. A small frequency shift instead of a scaling factor was applied to the theoretical IR spectra, specifically $8 \mathrm{~cm}^{-1}\left(\mathrm{Au}_{9}\right), 8 \mathrm{~cm}^{-1}\left(\mathrm{Au}_{10}\right), 12 \mathrm{~cm}^{-1}\left(\mathrm{Au}_{11}\right)$, $3 \mathrm{~cm}^{-1}\left(\mathrm{Au}_{12}\right)$, and $8 \mathrm{~cm}^{-1}\left(\mathrm{Au}_{13}\right)$ - see [55] and references within for discussion on this issue.

\section{B. Experimental}

Vibrational spectra are obtained by FIR-MPD spectroscopy of complexes of neutral gold clusters with $\mathrm{Kr}$ atoms. In short, the gas phase clusters are produced via laser ablation and thermalized to $\sim 100 \mathrm{~K}$. By adding $1.5 \% \mathrm{Kr}$ to the He carrier gas, formation of $\mathrm{Kr}$ complexes of the neutral gold clusters is achieved. By ionization with an $\mathrm{F}_{2}$ laser $(157 \mathrm{~nm}, 7.9 \mathrm{eV})$ the cluster distribution can be analyzed using time-of-flight mass spectrometry. Before reaching the mass spectrometer the neutral cluster beam is irradiated by light pulses from the Free Electron Laser for Infrared eXperiments (FELIX) [90]. If at a given IR frequency a cluster complex has an IR active mode, it can absorb photons, eventually leading to dissociation of the complex. This intensity change in the neutral cluster distribution is detected after ionization by the same relative intensity change in the mass spectrum. By scanning the frequency of FELIX, mass selective FIR-MPD spectra of the Kr-tagged gold clusters are obtained from the IR induced intensity change $I / I_{0}$ and plotted as experimental cross section $1 / P \ln I_{0} / I$ with $P$ being the IR laser power at a given wave number. More details on the experimental methods have been given previously [66].

\section{RESULTS AND DISCUSSION}

\section{A. Impact of density-functional approximations and van der Waals interactions on gold cluster stability}

Accurate first-principles predictions are needed to discern the stabilities of cluster isomers at finite temperatures. Thus, we first briefly discuss the impact of selecting various DFAs for predicting the relative energetics of low-energy planar and nonplanar structures for neutral $\mathrm{Au}_{5}-\mathrm{Au}_{13}$ clusters. We compare our DFA calculations relative to RPA. RPA is an attractive method for comparison because the RPA correlation energy is fully nonlocal and includes long-range $\mathrm{vdW}$ interactions and dynamic electronic screening, and the exact-exchange energy fully cancels the self-interaction error present in the Hartree energy [78]. Ultimately, the planar and nonplanar $\mathrm{Au}_{5}-\mathrm{Au}_{13}$ structures predicted to be most stable at $0 \mathrm{~K}$ are shown in Fig. 1.

Energy differences between the most stable nonplanar and planar isomers of $\mathrm{Au}_{5}-\mathrm{Au}_{13}$ (structures corresponding to Fig. 1) using various DFAs and RPA@PBE are shown in Fig. 2. Geometries were relaxed for each cluster with the respective DFA, but RPA@PBE was used as a single point correction on top of PBE-optimized geometries. Energetics corresponding to Fig. 2 are provided within Table S1 in the Supplemental Material [91]. The preference toward favoring
3D gold isomers over 2D isomers generally increases as cluster size increases. For $n=5-10$ and 12, all DFAs predict planar structures are the most stable, but for $n=11$ and 13 there are differences. The critical transition size from $2 \mathrm{D}$ to $3 \mathrm{D}$ at $0 \mathrm{~K}$ is above $\mathrm{Au}_{13}$ using PBE, whereas for all other DFAs and RPA@PBE it is $\mathrm{Au}_{11}$. The 2D to 3D critical transition size at $0 \mathrm{~K}$ was previously predicted at $\mathrm{Au}_{11}$, where $\mathrm{Au}_{11}$ preferred a capped-trigonal prism $\left(\mathrm{D}_{3 h}\right)$ structure [44,92], which is the same nonplanar isomer we found (Fig. 1). Although $\mathrm{Au}_{11}$ prefers a nonplanar isomer at $0 \mathrm{~K}$, all our DFA and RPA@PBE calculations predict $\mathrm{Au}_{12}$ to prefer a planar di-capped elongated hexagon $\left(\mathrm{D}_{3 h}\right)$. Another first-principles study also found $\mathrm{Au}_{12}$ prefers a planar di-capped elongated hexagon at $0 \mathrm{~K}$ [67], whereas other studies predict a nonplanar "cup" $\left(\mathrm{C}_{2 v}\right)$ structure as the preferred isomer [44]. As we discuss further below, the comparison of the experimental FIR-MPD spectra to theoretical IR spectra identifies the $\mathrm{Au}_{12}$ nonplanar cup isomer at $100 \mathrm{~K}$. PBE+MBD, HSE06+MBD, and RPA@PBE predict the same ground-state structure for $\mathrm{Au}_{5}-\mathrm{Au}_{12}$, but PBE+MBD slightly favors a planar $\mathrm{Au}_{13}$ isomer $\left(\Delta E_{3 \mathrm{D} \rightarrow 2 \mathrm{D}}=-34 \mathrm{meV}\right)$, whereas HSE06+MBD and RPA@PBE calculations favor a nonplanar $\mathrm{Au}_{13}$ structure. Indeed, $\mathrm{Au}_{13}$ has been suggested to be near the critical 2D to 3D transition size on multiple occasions, with much debate on the ground-state structure due to the sensitivity to the first-principles modeling approach $[26,27,37,44,45]$.

For the data in Fig. 2, the DFA error with respect to RPA@PBE increases monotonically with gold cluster size. HSE06+TS and HSE06+MBD agree best with RPA@PBE among our tested DFAs [mean absolute error (MAE) of 57 and $30 \mathrm{meV}$, respectively], but the computational cost of a hybrid functional prohibits their use in replica-exchange $a b$ initio molecular dynamics simulations. Nevertheless, the results of Fig. 2 show that HSE calculations are not needed for this paper. The good tradeoff between accuracy and computational cost of PBE+MBD justifies its use in our REMD calculations to compute the free energies of the structures of neutral $\mathrm{Au}_{5}-\mathrm{Au}_{13}$ clusters.

Interestingly, for $\mathrm{Au}_{5}-\mathrm{Au}_{9}$ all DFAs give essentially the same $\Delta E_{3 \mathrm{D} \rightarrow 2 \mathrm{D}}$, thus the non-vdW tail interactions favor the 2D structures. However, including long-range vdW interactions is important for larger clusters to improve agreement with RPA@PBE. HSE06 typically performs worse than $\mathrm{PBE}+\mathrm{TS}$ and $\mathrm{PBE}+\mathrm{MBD}$ with respect to RPA@PBE for predicting $\Delta E_{3 \mathrm{D} \rightarrow 2 \mathrm{D}}$ of $\mathrm{Au}_{5}-\mathrm{Au}_{13}$, having a MAE of $130 \mathrm{meV}$. Correcting PBE with MBD has a more pronounced effect on the relative isomer energetics than changing from PBE to HSE06. Going from PBE to PBE+TS or $\mathrm{PBE}+\mathrm{MBD}$ decreases the MAE with respect to RPA@PBE by 94 and $116 \mathrm{meV}$, respectively. Similarly, going from HSE6 to HSE06+TS or HSE06+MBD decreases the MAE with respect to RPA@PBE by 73 and $100 \mathrm{meV}$. The DFA+MBD scheme typically performs better than DFA+TS. This result is expected because the TS method simply computes the vdW energy using the ground-state electron density and includes hybridization effects for the polarizability but neglects longrange electrostatic screening and many-body contributions beyond pairwise interactions. However, the MBD scheme corrects the issues of TS by including the screened longrange many-body vdW energy via solution of the Schrödinger equation for a system of coupled electronic oscillators [76]. 


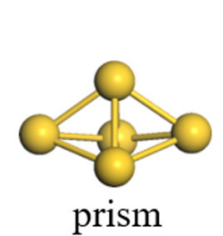

$\mathrm{Au}_{5}$
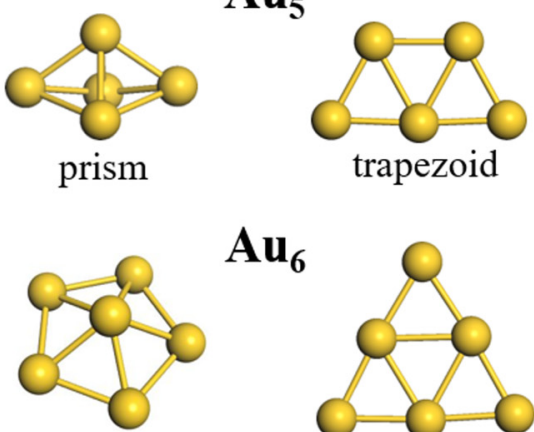

capped pentagon

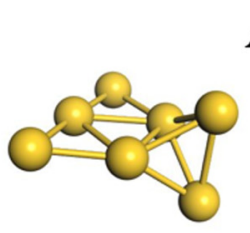

top-capped triangle
$\mathrm{Au}_{6}$

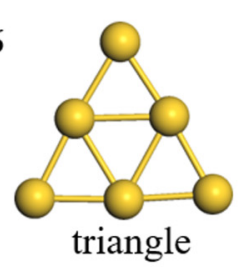

$\mathrm{Au}_{7}$

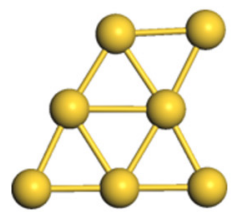

edge-capped triangle

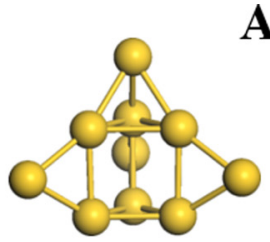

tetra-capped prism
$\mathrm{Au}_{10}$

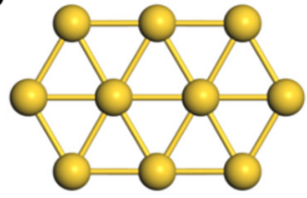

elongated hexagon
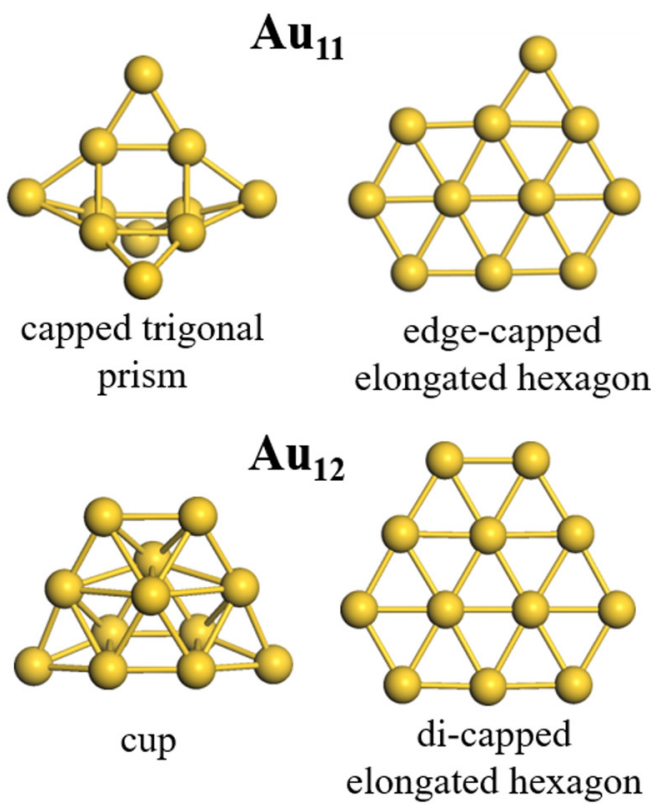

triangular pyramid

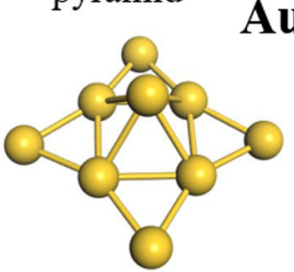

$\mathrm{Au}_{9}$

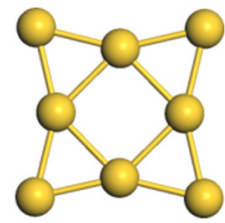

4-fold edge-capped square

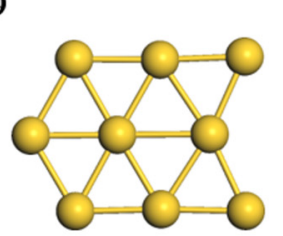

capped 4-fold- di-capped hexagon edge-capped square

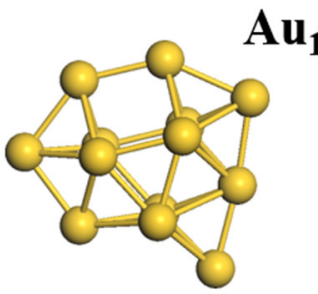

isomer 13-T

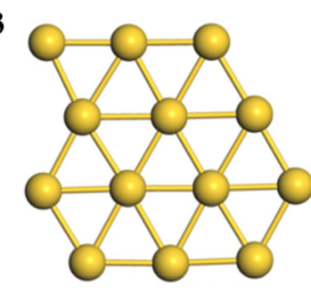

isomer $13-\mathrm{R}$

FIG. 1. Most stable planar and nonplanar gold cluster structures for $\mathrm{Au}_{5}-\mathrm{Au}_{13}$ at $0 \mathrm{~K}$. The same low-energy structures are identified for all considered density-functional approximations (e.g., using either PBE+MBD or HSE06+MBD) and RPA.

None of the vdW corrections applied in this paper is expected to yield a full account of vdW interactions among $\mathrm{Au}$ atoms [93], due to delocalized $6 s$ electrons, which challenge the charge partitioning scheme behind the adopted $\mathrm{vdW}$-correction methods. However, we note the remarkable quantitative agreement between the vdW-corrected DFAs, and in particular HSE06+MBD and RPA@PBE when the relative energy between 2D and 3D structures are compared (Fig. 2). This gives us confidence that the essence of the relative stability between planar and nonplanar $\mathrm{Au}_{n}$ structures is captured by the adopted vdW-correction schemes, in particular MBD.

Our results show that including long-range vdW interactions stabilizes 3D structures relative to 2D structures, especially as cluster size increases above seven atoms (Fig. 3). For example, using PBE+MBD the vdW interactions stabilize $3 \mathrm{D}$ structures relative to $2 \mathrm{D}$ structures by $110 \mathrm{meV}$ for $\mathrm{Au}_{8}$ and $456 \mathrm{meV}$ for $\mathrm{Au}_{13}$. Similar trends are found using HSE06+MBD and the TS scheme with and without self-consistent screening (SCS) to account for electrodynamic response effects.

We next predicted the average isotropic static polarizability per atom $\left(\alpha_{\text {iso }}\right)$ of the lowest-energy planar and nonplanar gold clusters as a function of size using HSE06+MBD(SCS) and HSE06+TS (Fig. 4). MBD(SCS) properly captures the static polarizability, whereas the TS scheme does not. There is an odd-even oscillation with respect to cluster size using MBD(SCS), where odd-sized gold clusters typically show larger polarizabilities than their even-sized neighbors as expected for "open/closed-shell systems". Also, we predict that 2D clusters have a larger $\alpha_{\text {iso }}$ than 3D clusters. Nevertheless, even though the 2D clusters have a higher average $\alpha_{\text {iso }}$, the atoms of the $3 \mathrm{D}$ clusters have higher atomic coordination (due to their more compact nature), leading to stronger stabilization by vdW forces (Figs. S1 and S2 in [91]). 


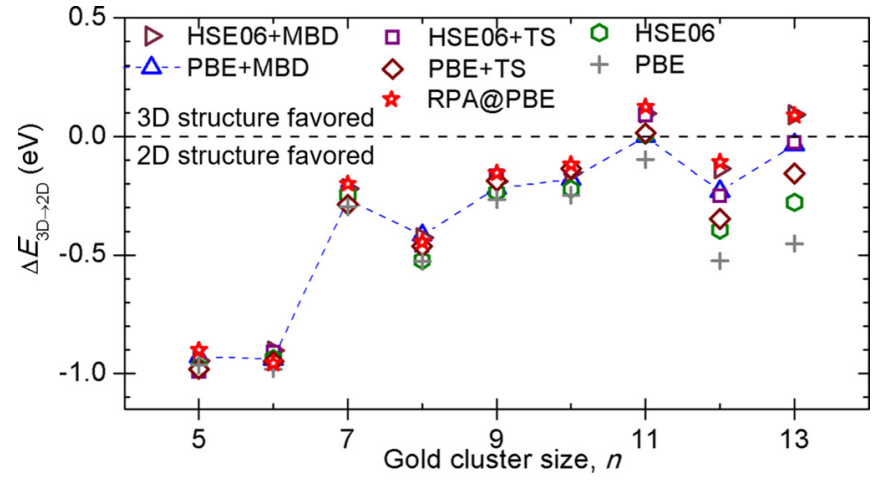

FIG. 2. Energy difference between the lowest-energy nonplanar and planar isomers $\left(\Delta E_{3 \mathrm{D} \rightarrow 2 \mathrm{D}}\right)$ for $\mathrm{Au}_{5}-\mathrm{Au}_{13}$ using various densityfunctional approximations and beyond at $0 \mathrm{~K}$. The dashed blue line is used to guide the eye for the PBE+MBD results. TS, TkatchenkoScheffler pairwise van der Waals correction; MBD, many-body dispersion with range separated self-consistent screening; RPA@PBE, random-phase approximation using PBE orbitals; 3D, nonplanar; 2D, planar.

\section{B. Free-energy surfaces of $\mathrm{Au}_{5}-\mathrm{Au}_{13}$ : Impact of temperature on cluster isomer stability}

The usual modeling approach to probe the stability of cluster isomers is to compare the energies of various isomers at $0 \mathrm{~K}$. However, experiments are performed at finite temperatures, thus structures with low free energy (high Boltzmann probability) should be determined. Calculating free energy of clusters, which include anharmonic contributions and the effect of frequent structural rearrangements (fluxionality), requires sampling many configurations to capture the enthalpic and entropic contributions to the free energy. Our REMD+MBAR simulations efficiently sampled the potentialenergy surfaces of neutral $\mathrm{Au}_{n}$ clusters $(n=5-13)$ without requiring knowledge a priori and let us compute free energies of gold cluster isomers, and hence their Boltzmann proba-

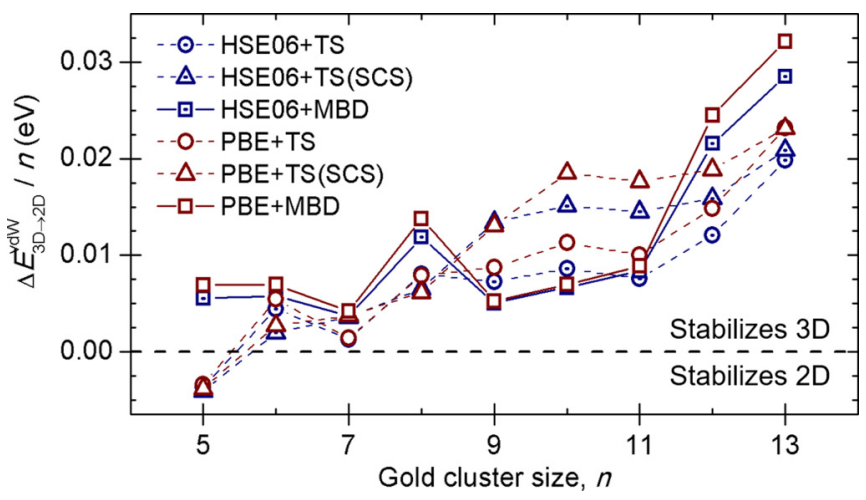

FIG. 3. Difference in van der Waals energy per atom $\left(\Delta E^{\mathrm{vdW}}{ }_{3 \mathrm{D} \rightarrow 2 \mathrm{D}} / n\right)$ between the most stable planar and nonplanar isomers of $\mathrm{Au}_{5}-\mathrm{Au}_{13}$ at $0 \mathrm{~K}$ using various density-functional approximations and van der Waals corrections. TS, Tkatchenko-Scheffler pairwise van der Waals; TS(SCS), Tkatchenko-Scheffler pairwise van der Waals with self-consistent screening; MBD, many-body dispersion with range separated self-consistent screening; 3D, nonplanar; 2D, planar.

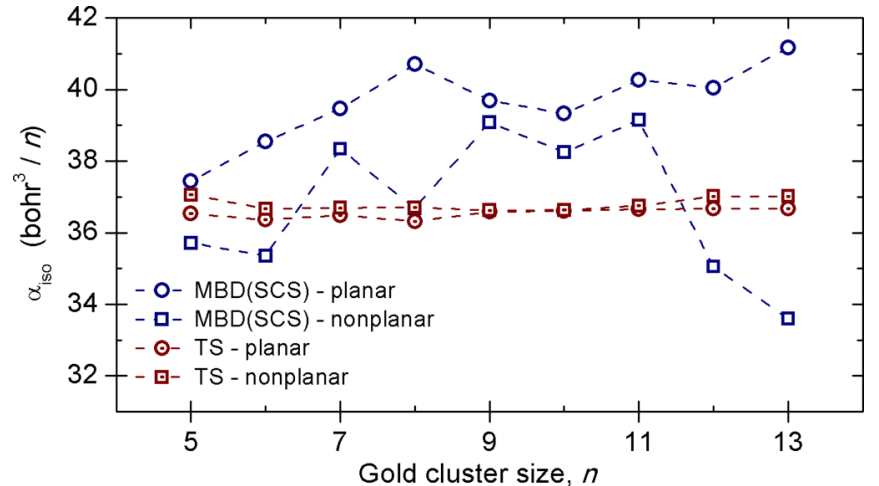

FIG. 4. Average isotropic static polarizability $\left(\alpha_{\text {iso }}\right)$ per atom of the lowest-energy planar and nonplanar gold clusters as a function of size predicted using HSE06+MBD(SCS) and HSE06+TS. The planar and nonplanar gold clusters correspond to those shown in Fig. 1. TS, Tkatchenko-Scheffler pairwise van der Waals; MBD(SCS), many-body dispersion with self-consistent screening.

bility. Gold cluster isomers and their Boltzmann populations are depicted in two-dimensional free-energy surfaces using two order parameters that were selected a posteriori, namely, the gold cluster's "coordination similarity" and "radius of gyration." Together, these two order parameters allow one to discern the different planar and nonplanar isomers in the simulation. These two order parameters can also discern between slightly distorted planar structures (due to out-ofplane vibrational modes at finite temperature) and nonplanar structures. The coordination similarity order parameter is obtained by constructing a coordination histogram (distribution of Au-atoms coordination number, i.e., number of $\mathrm{Au}$ atoms bonded to each $\mathrm{Au}$ atom) for each cluster configuration [94] and computing the cosine distance between each coordination histogram with respect to the ground-state reference (each cluster configuration referred to the ground state at the same size). Example bond coordination histograms for $\mathrm{Au}_{8}$ at 100 and $300 \mathrm{~K}$ are provided in Fig. S2 in [91]. Radius of gyration is computed by taking the root-mean-square distance of the cluster's atoms with respect to its center of mass. Note, point groups of clusters are assigned based on their optimized structures at $0 \mathrm{~K}$.

\section{1. $\mathrm{Au}_{5}-\mathrm{Au}_{8}$}

Our free-energy favored structures fully agree with previous assignments for $\mathrm{Au}_{5}, \mathrm{Au}_{6}, \mathrm{Au}_{7}$, and $\mathrm{Au}_{8}$ based on FIRMPD spectroscopy experiments (at $100 \mathrm{~K}$ ) and ground-state DFT calculations $[66,95] . \mathrm{Au}_{5}$ is most stable as a trapezoid $\left(\mathrm{C}_{2 v}\right)$, having a Boltzmann probability $P(T)$ of $P(100 \mathrm{~K})=$ $100 \%$ and $P(300 \mathrm{~K})=99.2 \%$ (Fig. S3 in [91]). The only thermodynamically competing structure at $300 \mathrm{~K}$ is the bowtie $\left(\mathrm{D}_{2 h}\right) . \mathrm{Au}_{6}$ prefers a planar triangle $\left(D_{3 h}\right)$ structure with $\mathrm{P}(100 \mathrm{~K})=100 \%$ and $P(300 \mathrm{~K})=100 \%$ according to the free-energy calculations (Fig. S4 in [91]). The $\mathrm{Au}_{7}$ cluster adopts the well-known edge-capped triangle $\left(C_{s}\right)$ with $P(100$ $\mathrm{K})=100 \%$ and $P(300 \mathrm{~K})=99.3 \%$, with the second most likely structure being a hexagon $\left(\mathrm{D}_{6 h}\right)$ with $P(300 \mathrm{~K})=0.2 \%$ (Fig. S5 in [91]). The remaining $0.5 \%$ of the $\mathrm{Au}_{7}$ population is an assortment of other structures, indicative of the fluxional 


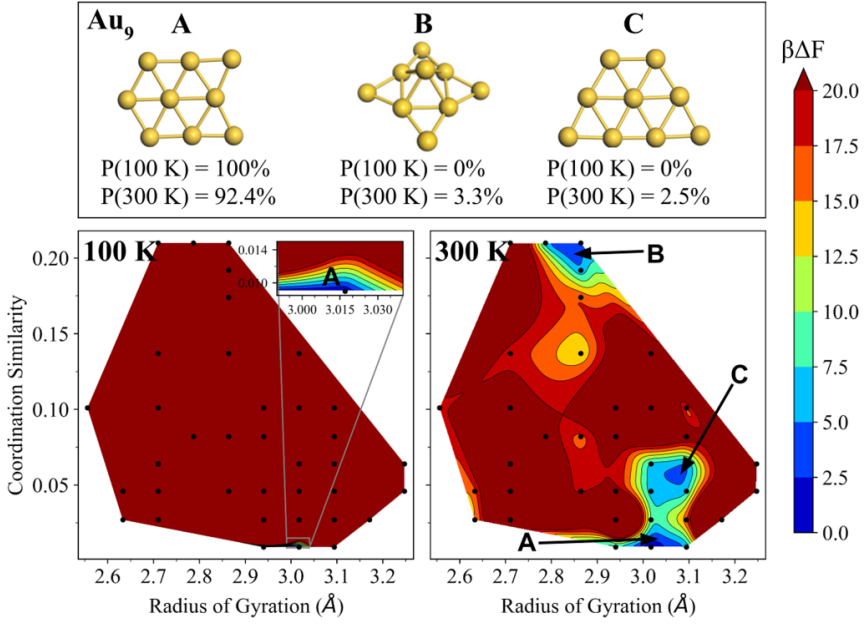

FIG. 5. Free-energy surfaces of $\mathrm{Au}_{9}$ at $100 \mathrm{~K}$ (left) and $300 \mathrm{~K}$ (right). The dominant isomer 9-A (di-capped hexagon, $\mathrm{C}_{2 v}$ ) and competing isomers 9-B (capped fourfold-edge-capped square, $\mathrm{C}_{4 v}$ ) and 9-C (trapezoid, $\mathrm{C}_{2 v}$ ) have their Boltzmann probabilities $P(T)$ specified at each temperature, as well as their locations marked on the free-energy surfaces. The color bar represents dimensionless free-energy values $(\beta \Delta F)$, where $\beta$ is $1 / k_{B} T$. Dark red regions have $\beta \Delta F \geqslant 20$. The black points indicate sampled cluster geometries having the binned (coordination similarity, radius of gyration) value. $\mathrm{A}$ is used as the reference state for coordination similarity, thus its coordination similarity is 0.0 .

behavior of this system at $300 \mathrm{~K}$. Nevertheless, for $\mathrm{Au}_{5}-\mathrm{Au}_{7}$ the impact of temperature on the isomer population is quite negligible up to $300 \mathrm{~K}$, justifying the typical 0 -K modeling approach; however, for $\mathrm{Au}_{8}$ the impact of temperature at $300 \mathrm{~K}$ is already quite noticeable. In agreement with past FIR-MPD experiments at $100 \mathrm{~K} \mathrm{[66]} \mathrm{and} \mathrm{computational} \mathrm{predictions} \mathrm{at}$ $0 \mathrm{~K}$ [43], our free-energy calculations predict $\mathrm{Au}_{8}$ prefers a planar fourfold edge-capped square $\left(\mathrm{D}_{4 h}\right)$ structure with $P(100 \mathrm{~K})=100 \%$ (Fig. S6 in [91]). Yet, at $300 \mathrm{~K}$ there is a competing nonplanar $\mathrm{Au}_{8}$ isomer (triangular pyramid, $\left.\mathrm{C}_{3 v}\right)$ with $P(300 \mathrm{~K})=5.4 \%$. Thus, even for clusters as small as $\mathrm{Au}_{8}$ there can be a thermodynamically competing 3D isomer with an appreciable Boltzmann probability at room temperature. As we show below, the impact of raising temperature above $100 \mathrm{~K}$ typically stabilizes nonplanar gold cluster structures over planar structures, except for $\mathrm{Au}_{11}$.

The perfect agreement between the here predicted structures of $\mathrm{Au}_{5}-\mathrm{Au}_{8}$ at $100 \mathrm{~K}$ and previous structural assignments from experimental FIR-MPD spectra at $100 \mathrm{~K}$ gives us confidence in our REMD+MBAR modeling approach using PBE+MBD. In the remainder of this section we analyze the free-energy surfaces of $\mathrm{Au}_{9}-\mathrm{Au}_{13}$ to examine isomer stability at finite temperatures. In the next section we compare the freeenergy favored structures of $\mathrm{Au}_{9}-\mathrm{Au}_{13}$ with experimentally measured and theoretically predicted IR spectra to finally assign the structures of $\mathrm{Au}_{9}-\mathrm{Au}_{13}$ at $100 \mathrm{~K}$.

\section{2. $\mathrm{Au}_{9}$}

The free-energy surface of $\mathrm{Au}_{9}$ shown in Fig. 5 predicts the most stable isomer at both 100 and $300 \mathrm{~K}$ is a di-capped hexagon with $\mathrm{C}_{2 v}$ symmetry (isomer 9-A)

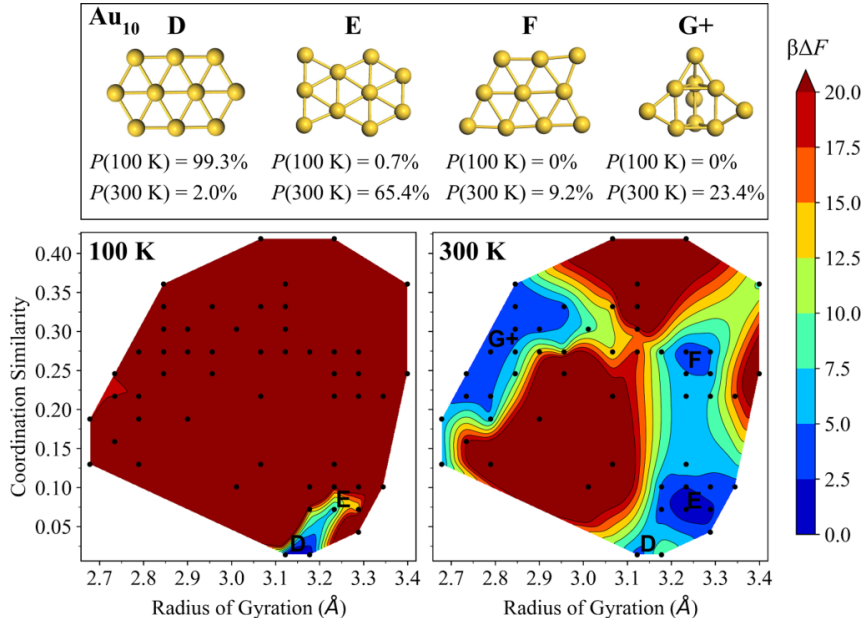

FIG. 6. Free-energy surfaces of $\mathrm{Au}_{10}$ at $100 \mathrm{~K}$ (left) and $300 \mathrm{~K}$ (right). The dominant cluster isomers 10-D (elongated hexagon, $\mathrm{D}_{2 h}$ ), 10-E (tri-capped hexagon, $C_{2 h}$ ), 10-F (capped trapezoid, $\mathrm{C}_{s}$ ), and 10-G+ have their Boltzmann probabilities $P(T)$ specified at each temperature, as well as their locations marked on the free-energy surface.

with $P_{9-\mathrm{A}}(100 \mathrm{~K})=100 \%$ and $P_{9-\mathrm{A}}(300 \mathrm{~K})=92.4 \%$. Previous first-principles calculations also predicted $9-\mathrm{A}$ to be the ground-state structure at $0 \mathrm{~K}$ [44]. Isomer 9-A is clearly identified as the preferred $\mathrm{Au}_{9}$ structure at $100 \mathrm{~K}$ according to our FIR-MPD spectroscopy measurements and theoretical IR spectra (discussed further below, Fig. 10). At $300 \mathrm{~K}$, however, there are two competing isomers $9-\mathrm{B}$ and $9-\mathrm{C}$ with nonnegligible Boltzmann probabilities of $P_{9-\mathrm{B}}(300 \mathrm{~K})=3.3 \%$ and $P_{9-\mathrm{C}}(300 \mathrm{~K})=2.5 \%$. The nonplanar $9-\mathrm{B}$ is a capped fourfold edge-capped square (built off the most stable $\mathrm{Au}_{8}$ structure), whereas $9-\mathrm{C}$ is a planar trapezoid. Isomers 9-A and 9-C, having simply a different position of one periphery (edge) gold atom, have a more similar radius of gyration and coordination similarity compared with 9-B. Although nonplanar isomers are thermodynamically stable for $\mathrm{Au}_{9}$ even at $300 \mathrm{~K}$, the barriers between the planar (9-A and 9-C) and nonplanar (9-B) isomers are calculated to be much greater than $25 \beta \Delta F(0.65 \mathrm{eV})$, thus $\mathrm{Au}_{9}$ might be kinetically trapped in planar or nonplanar structures.

\section{3. $\mathrm{Au}_{10}$}

The free-energy surface of $\mathrm{Au}_{10}$ shown in Fig. 6 predicts the most stable isomer at $100 \mathrm{~K}$ is an elongated hexagon with $\mathrm{D}_{2 h}$ symmetry (10-D) with $P_{10-\mathrm{D}}(100 \mathrm{~K})=99.3 \%$. Previous first-principles calculations at $0 \mathrm{~K}$ also predicted 10-D as the ground-state structure [44]. Moreover, 10-D is identified as the preferred $\mathrm{Au}_{10}$ structure at $100 \mathrm{~K}$ according to the assignment of the experimental FIR-MPD spectra (discussed further below, Fig. 10). However, there are multiple competing planar (10-E, 10-F) and nonplanar (10-G+) isomers at $300 \mathrm{~K}$. Interestingly, the most stable isomer $10-\mathrm{D}$ at $100 \mathrm{~K}$ is not the most stable isomer at $300 \mathrm{~K}$, which is instead predicted to be 10-E. Judging from the width and shallow nature of the freeenergy basin of 10-E, the stability of $10-\mathrm{E}$ over $10-\mathrm{D}$ at elevated temperature may arise from a larger configurational and vibrational contribution to the free energy. Additionally, there 


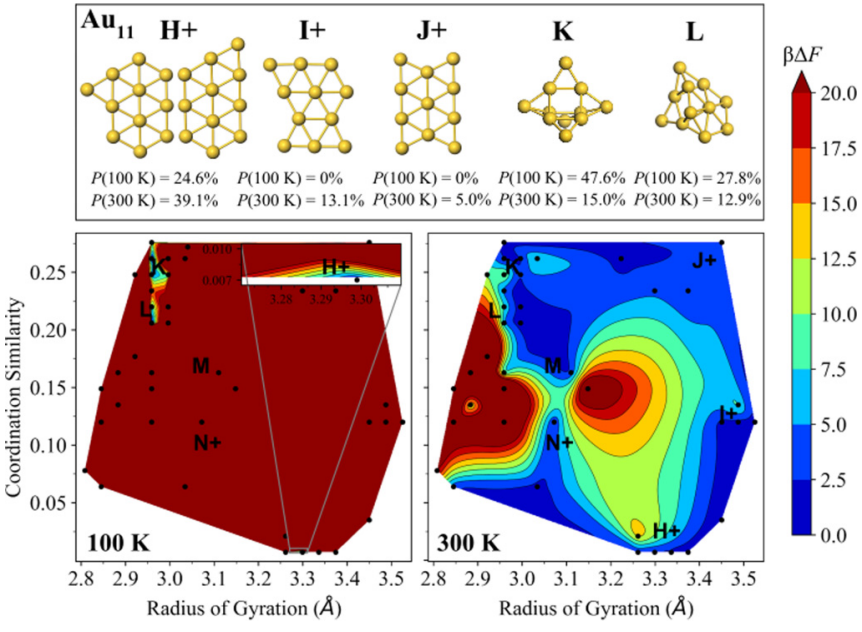

FIG. 7. Free-energy surfaces of $\mathrm{Au}_{11}$ at $100 \mathrm{~K}$ (left) and $300 \mathrm{~K}$ (right). The dominant cluster isomers 11-H+, 11-I+, 11-J+, 11-K, and 11-L have their Boltzmann probabilities $P(T)$ specified at each temperature, as well as their locations marked on the free-energy surface. Isomers with $P(300 \mathrm{~K})<1 \%$ are not shown. Structures M, $\mathrm{N}+$, and $\mathrm{I}+$ are given in Fig. S9 in [91].

are also three competing nonplanar isomers (10-G+) at $300 \mathrm{~K}$ with a combined probability of $P_{10-\mathrm{G}+}(300 \mathrm{~K})=23.4 \%(\mathrm{G}+$ structures shown in Fig. S7 in [91]). These three structures are not readily distinguishable by our order parameters, all having similar radius of gyration and coordination similarity, thus they are lumped into a single state. The broad and shallow free-energy basin of the nonplanar isomers $10-\mathrm{G}+$ suggests these structures can readily interconvert at room temperature, whereas the free-energy barrier to interconvert between planar and nonplanar isomers is likely much higher. At elevated temperatures of 500 and $750 \mathrm{~K}$, the various $\mathrm{Au}_{10}$ planar and nonplanar isomers dynamically coexist as kinetic barriers can be readily overcome (Fig. S8 in [91]).

\section{4. $\mathrm{Au} \mathbf{u}_{11}$}

For $\mathrm{Au}_{11}$, several isomers, planar $11-\mathrm{H}+$ (differently edgecapped elongated hexagons) as well as nonplanar $11-\mathrm{K}$ (capped trigonal prism) and 11-L have non-negligible Boltzmann probabilities at $100 \mathrm{~K}$ (Fig. 7). At $300 \mathrm{~K}$, a variety of planar and nonplanar $\mathrm{Au}_{11}$ structures can coexist, with roughly $P(300 \mathrm{~K})=55 \%$ for observing a planar structure. The metastable isomers (11-I+, 11-M, and 11-N+) not shown in Fig. 7 are shown in Fig. S9 in [91]. The ensemble of planar structures of $\mathrm{Au}_{11}$ resembles the planar structures of $\mathrm{Au}_{10}$, except with an 11 th atom that can easily migrate around the cluster periphery to adopt a variety of distinct and stable planar isomers. The nonplanar $11-\mathrm{K}$ has the lowest free energy at $100 \mathrm{~K}$ and is identified according to our experimental FIRMPD and theoretical IR spectra (discussed below, Fig. 10), indicating that $\mathrm{Au}_{11}$ is the critical 2D/3D transition size at $100 \mathrm{~K}$.

\section{5. $\mathrm{Au}_{12}$}

Santarossa et al. analyzed the free-energy surface of $\mathrm{Au}_{12}$ via metadynamics using $\mathrm{PBE}+\mathrm{Grimme}-\mathrm{D} 1$ and found

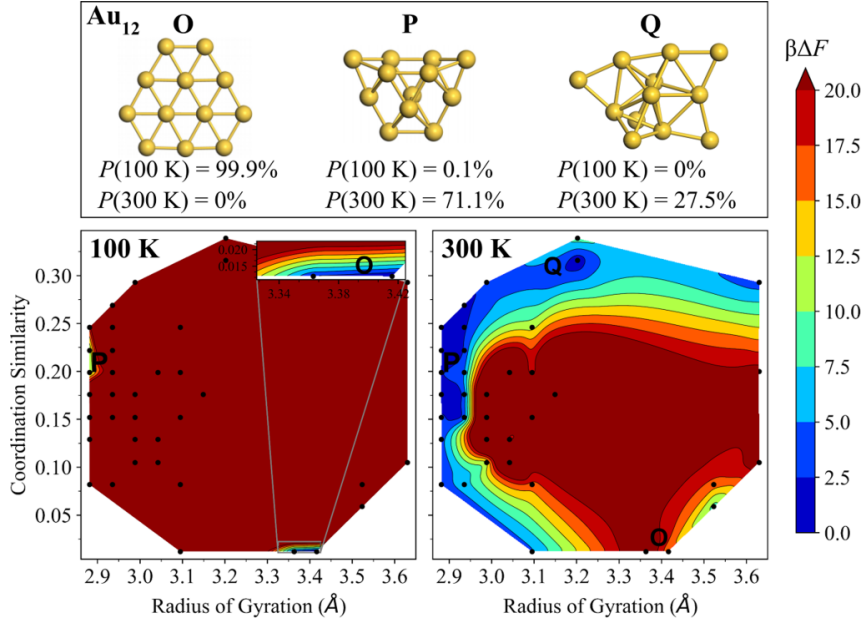

FIG. 8. Free-energy surfaces of $\mathrm{Au}_{12}$ at $100 \mathrm{~K}$ (left) and $300 \mathrm{~K}$ (right). The dominant cluster isomers 12-O (di-capped elongated hexagon, $\mathrm{D}_{3 h}$ ), 12-P (cup, $\mathrm{C}_{2 v}$ ), and 12-Q $\left(C_{s}\right)$ have their Boltzmann probabilities $P(T)$ specified at each temperature, as well as their locations marked on the free-energy surfaces. Isomers with $P(300 \mathrm{~K})<1 \%$ are not shown.

a nearly equal probability for $2 \mathrm{D}$ and $3 \mathrm{D}$ isomers at room temperature, whereas the lowest-energy $2 \mathrm{D}$ isomer is $0.2 \mathrm{eV}$ more stable than the lowest-energy $3 \mathrm{D}$ structure at $0 \mathrm{~K} \mathrm{[58].}$ In contrast, our free-energy calculations predict the nonplanar isomers will dominate at room temperature (Fig. 8). The most stable planar (12-O) and nonplanar (12-P) isomers we predict match those of $[2,44,67]$ at $0 \mathrm{~K}$; however, Santarossa et al. predicted 12-O and 12-P to be 0.65 and $1.64 \mathrm{eV}$ uphill from the global minima, respectively [58]. Although at $100 \mathrm{~K}$ the planar di-capped elongated hexagon (12-O) is most stable with $P_{12-\mathrm{O}}(100 \mathrm{~K})=99.9 \%$, at $300 \mathrm{~K}$ the nonplanar $12-\mathrm{P}$ and $12-\mathrm{Q}$ have $P_{12-\mathrm{P}}(300 \mathrm{~K})=71.1 \%$ and $P_{12-\mathrm{Q}}(300 \mathrm{~K})=$ $27.5 \%$, respectively.

\section{6. $\mathrm{Au}_{13}$}

Using $\mathrm{PBE}+\mathrm{MBD}$, we find that $\mathrm{Au}_{13}$ at $100 \mathrm{~K}$ prefers a planar structure with $P(100 \mathrm{~K})=86.2 \%$, although a variety of nonplanar structures has appreciable probabilities (Fig. S10 in [91]). Although a few planar $\mathrm{Au}_{13}$ isomers have a similar total energy to that of the lowest-energy nonplanar $\mathrm{Au}_{13}$ isomers, they were rarely seen due to temperature effects at $300 \mathrm{~K}$. At $300 \mathrm{~K}$ a variety of nonplanar isomers are preferred with a cumulative probability of $P(300 \mathrm{~K})=90.5 \%$. Indeed, lowenergy isomers of $\mathrm{Au}_{13}$ are known to be disordered, forming a nearly continuous distribution of nonplanar structures as a function of potential energy $[50,57,96]$.

The free-energy surface can be calculated at any temperature within our REMD temperature-simulation window using MBAR. By integrating the free-energy surfaces of $\mathrm{Au}_{5}-\mathrm{Au}_{13}$, we show the impact of raising temperature from 100 to $400 \mathrm{~K}$ on the Boltzmann population of planar structures versus nonplanar structures (Fig. 9). Small gold clusters of neutral $\mathrm{Au}_{5}-\mathrm{Au}_{7}$ remain predominantly planar even as temperature is raised from 100 at $400 \mathrm{~K}$. However, increasing temperature from 100 to $400 \mathrm{~K}$ typically stabilizes 3D structures over 2D isomers for $\mathrm{Au}_{8}-\mathrm{Au}_{13}$, except for $\mathrm{Au}_{11}$ due to its 


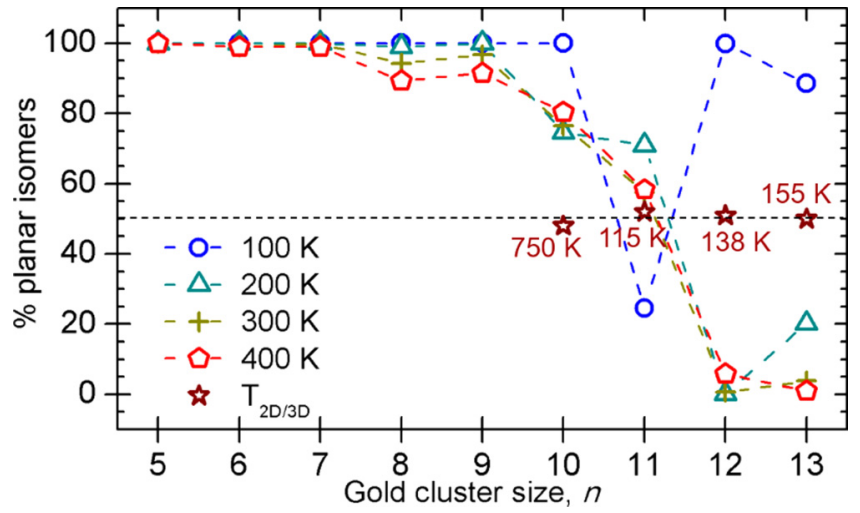

FIG. 9. Boltzmann population of planar isomers for $\mathrm{Au}_{5}-\mathrm{Au}_{13}$ as a function of temperature $(100-400 \mathrm{~K})$ based on free-energy calculations. $T_{2 \mathrm{D} / 3 \mathrm{D}}$ corresponds to the temperature where there are roughly 50\% planar and 50\% nonplanar structures.

large number of nearly energy degenerate $2 \mathrm{D}$ structures that become kinetically accessible at elevated temperatures. Small populations of $3 \mathrm{D}$ isomers arise for $\mathrm{Au}_{8}$ and $\mathrm{Au}_{9}$ at $300 \mathrm{~K}$ and above ( $\sim 5$ and $10 \%$ at 300 and $400 \mathrm{~K}$, respectively).

For $\mathrm{Au}_{10}-\mathrm{Au}_{13}$, we identified the temperature $T_{2 \mathrm{D} / 3 \mathrm{D}}$ where there are roughly $50 \%$ planar and $50 \%$ nonplanar isomers. For $\mathrm{Au}_{10}$ at $\mathrm{T}_{2 \mathrm{D} / 3 \mathrm{D}}=750 \mathrm{~K}$, we compute a $48 \% \pm 13 \%$ population of planar structures (error bars are based on uncertainty propagation of the free energies and neglecting correlations across free-energy bins). Although $\mathrm{Au}_{11}$ slightly prefers a nonplanar structure over a planar structure at 100 $\mathrm{K}$, a $52 \% \pm 13 \%$ population of planar structures is calculated at $T_{2 \mathrm{D} / 3 \mathrm{D}}=115 \mathrm{~K} . \mathrm{Au}_{12}$ and $\mathrm{Au}_{13}$ adopt a $51 \% \pm 12 \%$ and $50 \% \pm 6.3 \%$ population of planar structures at 138 and $155 \mathrm{~K}$, respectively. The temperature where $\mathrm{Au}_{5}-\mathrm{Au}_{9}$ adopt roughly $50 \%$ planar structures is outside of our simulated temperature range (e.g., $\mathrm{Au}_{8}$ and $\mathrm{Au}_{9}$ only reach $\sim 65 \%$ planar structures at $1000 \mathrm{~K}$ ) and may not be achievable due to fragmentation at more elevated temperatures, i.e., there is effectively no planar/nonplanar transition for $\mathrm{Au}_{5}-\mathrm{Au}_{9}$. These results highlight that the critical transition size from planar to nonplanar is gradual and depends on temperature.

\section{Structural assignment of $\mathrm{Au}_{9}-\mathrm{Au}_{13}$ via far-IR spectroscopy}

Most prior experimental studies on the structure of gas phase gold clusters examined charged clusters due to the ease of size selection. Here we assign the structure of neutral $\mathrm{Au}_{9}-\mathrm{Au}_{13}$ clusters using experimental FIR-MPD spectroscopy and comparison to theoretical anharmonic IR spectra and computed free energies. FIR-MPD spectroscopy is a verified experimental technique for obtaining the vibrational spectra of metal clusters in the gas phase $[97,98]$. Some of the authors have previously structurally assigned the neutral $\mathrm{Au}_{3}, \mathrm{Au}_{4}, \mathrm{Au}_{5}, \mathrm{Au}_{7}, \mathrm{Au}_{8}, \mathrm{Au}_{19}$, and $\mathrm{Au}_{20}$ clusters using FIRMPD spectroscopy $[55,66,95]$.

In most cases, the experimental FIR-MPD spectra of metal clusters were interpreted only by comparison to calculated harmonic IR spectra at $0 \mathrm{~K}$. Experiments, however, are performed at finite temperature, and even at low temperatures anharmonic effects can impact the vibrational spectra by chang- ing the position of peaks, their intensities, and broadening, and can even cause the appearance of new peaks. Here, theoretical anharmonic IR spectra (from here on simply called theoretical IR spectra) of $\mathrm{Au}_{9}-\mathrm{Au}_{13}$ at $100 \mathrm{~K}$ are computed using ab initio molecular dynamics, which properly includes vibrational anharmonicity, to compare with experimental FIR-MPD spectra obtained at $100 \mathrm{~K}$. Figures 10 and 11 show the experimental FIR-MPD and theoretical IR spectra for $\mathrm{Au}_{9}-\mathrm{Au}_{13}$. Here we note possible sources of discrepancy between the experimental FIR-MPD spectra and the theoretical IR spectra. First, the experimental FIR-MPD spectra may only be sampling the colder part of the canonically distributed population of neutral gold clusters due to spontaneous dissociation of the $\mathrm{Au}_{n} \mathrm{Kr}_{m}$ complexes from the hotter tail of the canonical distribution [55]. Second, it is possible for fragmentation of $\mathrm{Au}_{n} \mathrm{Kr}_{m}$ into $\mathrm{Au}_{n} \mathrm{Kr}_{m-1}$ complexes to occur, which would affect the band intensities for $\mathrm{Au}_{n} \mathrm{Kr}_{m-1}$ [99]. Also, only the pure metal clusters are considered in the calculations because it can be assumed that the weak interaction between $\mathrm{Kr}$ and the larger Au clusters does not have a large impact on the IR spectra [55].

Johansson et al. predicted using DFT that the planar $\mathrm{Au}_{9}$ di-capped hexagon, the planar $\mathrm{Au}_{10}$ elongated hexagon, and the nonplanar $\mathrm{Au}_{11}$ capped-trigonal prism are the ground-state structures at $0 \mathrm{~K}$ [44]. These same $\mathrm{Au}_{9}-\mathrm{Au}_{11}$ structures are assigned at $100 \mathrm{~K}$ based on comparison of our free-energy calculations and theoretical IR spectra with experimental FIRMPD spectroscopy (Fig. 10).

\section{1. $\mathrm{Au}_{9}$}

The $\mathrm{Au}_{9}$ di-capped hexagon (9-A) is predicted to be the most probable at $100 \mathrm{~K}\left[P_{9-\mathrm{A}}(100 \mathrm{~K})=100 \%\right]$ and also has the best theoretical IR spectrum agreement with FIR-MPD measurements for $\mathrm{Au}_{9} \mathrm{Kr}_{2}$ (Fig. 10) according to the Pendry $R$ factor $\left(R_{P}=0.80\right)$. All other competing $\mathrm{Au}_{9}$ isomers have $0 \%$ Boltzmann probabilities at $100 \mathrm{~K}$ and worse theoretical IR spectra agreement based on Pendry analysis. Albeit lacking the fine structure of the theoretical anharmonic IR spectra, the theoretical harmonic IR spectra of $\mathrm{Au}_{9}$ (Fig. S11 in [91]) also supports this structural assignment. Note that the experimental spectrum of $\mathrm{Au}_{9} \mathrm{Kr}$ shows in comparison to $\mathrm{Au}_{9} \mathrm{Kr}_{2}$ some broader less resolved bands around $180 \mathrm{~cm}^{-1}$. This is probably due to a fragmentation of $\mathrm{Au}_{9} \mathrm{Kr}_{2}$ into $\mathrm{Au}_{9} \mathrm{Kr}$ affecting the band intensities for $\mathrm{Au}_{9} \mathrm{Kr}$. Alternatively one could assume that in $\mathrm{Au}_{9} \mathrm{Kr}$ a different structural isomer prevails, i.e., the rare gas binding may be isomer selective as seen for other gold clusters previously [99]. Comparison to the theoretical IR spectra of other isomers, however, does not give indications for the presence of a different isomer.

\section{2. $\mathrm{Au}_{10}$}

For $\mathrm{Au}_{10}$, there is no significant difference between the FIR-MPD spectra of the complexes with a single $\mathrm{Kr}$ atom and two $\mathrm{Kr}$ atoms, and Pendry analysis between the FIRMPD spectra and theoretical IR spectra indicates that either a nonplanar structure $\left(10-\mathrm{G}, R_{P}=0.80\right)$ or the planar elongated hexagon is preferred $\left(10-\mathrm{D}, R_{P}=0.81\right)$. The theoretical IR spectrum of the $10-\mathrm{G}$ nonplanar isomer, however, 

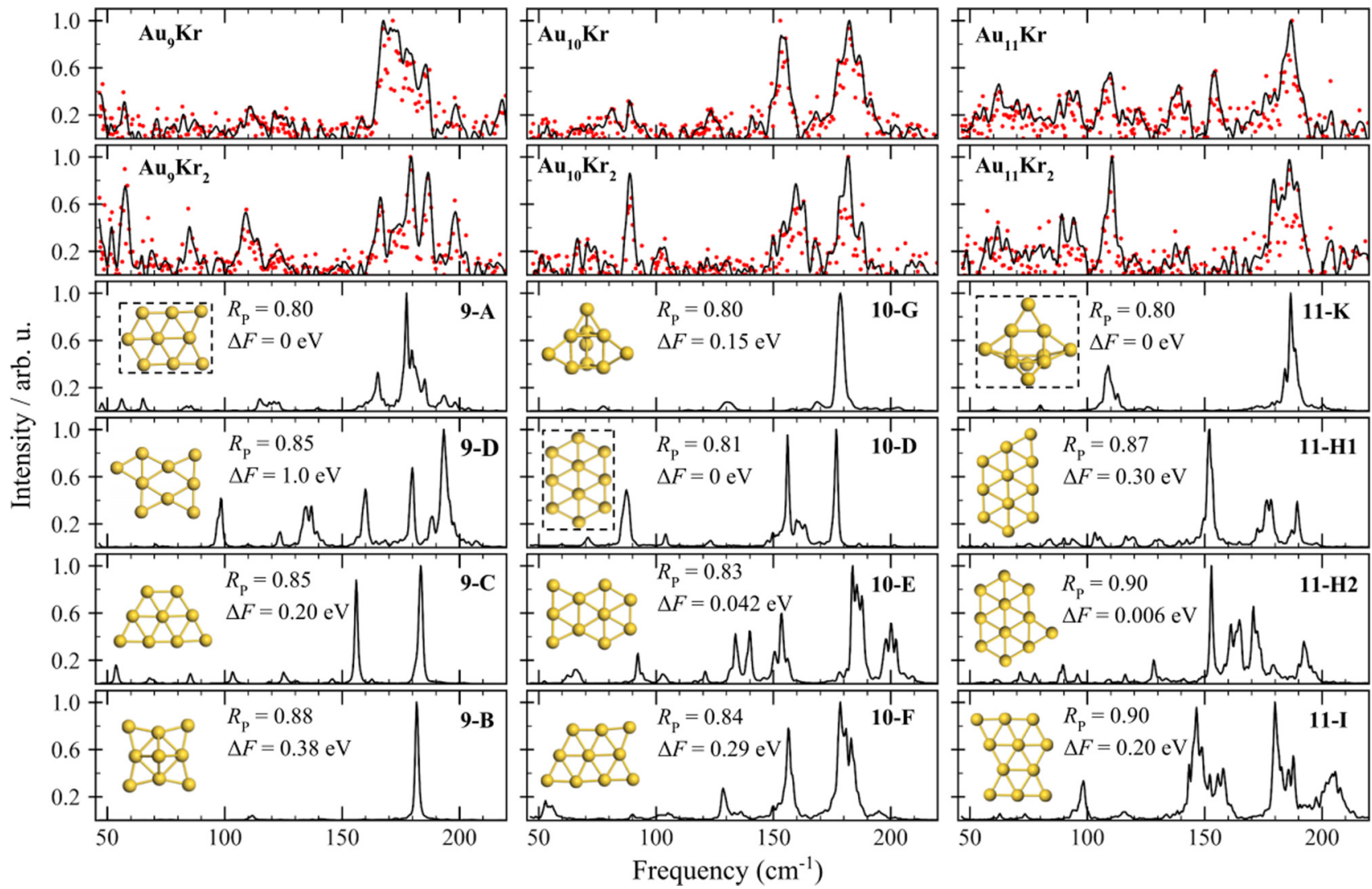

FIG. 10. Comparison of experimental FIR-MPD and theoretical anharmonic IR spectra for $\mathrm{Au}_{9}, \mathrm{Au}_{10}$, and $\mathrm{Au}_{11}$ at $100 \mathrm{~K}$. For each size, the two upper panels show the experimental spectra for the complexes with one and two $\mathrm{Kr}$ atoms and below theoretical IR spectra are shown. Pendry $R$ - factors $\left(R_{P}\right)$ comparing theoretical IR spectra with experimental results from $\mathrm{Au}_{9} \mathrm{Kr}_{2}, \mathrm{Au}_{10} \mathrm{Kr}$ or $\mathrm{Au}_{11} \mathrm{Kr}_{2}$ are indicated. Free-energy calculations ( $\Delta F$, referenced to the lowest free-energy isomer) are shown at $100 \mathrm{~K}$ for each isomer. Structures surrounded by a black-dashed box are assigned to experimental FIR-MPD spectra based on $R_{P}$ and the free-energy calculations. The black lines in the experimental FIR-MPD spectra are smoothed spectra, and the red dots are the raw data.

is missing prominent peaks seen in the experiment at $\sim 90$ and $\sim 160 \mathrm{~cm}^{-1}$, which are present in the theoretical IR spectrum of 10-D and the FIR-MPD spectra of $\mathrm{Au}_{10} \mathrm{Kr}$ and $\mathrm{Au}_{10} \mathrm{Kr}_{2}$. This highlights a weakness of the Pendry analysis, which heavily weights peak positions over peak intensities. Thus, it is also important to also consider the free energy of the complexes beyond $R_{P}$ to aid structural assignments. The nonplanar $10-\mathrm{G}$ structure is predicted to have a $0 \%$ Boltzmann probability at $100 \mathrm{~K}$. The planar 10-D has the lowest calculated free energy at $100 \mathrm{~K}$ and has the secondbest agreement with the FIR-MPD spectra according to $R_{P}$. Thus, for $\mathrm{Au}_{10}$ we assign the FIR-MPD spectra to the planar elongated hexagon (10-D). Based on this result, one may assign $\mathrm{Au}_{10}$ as the largest neutral gold cluster having a planar structure at $100 \mathrm{~K}$; however, one must keep in mind that the experiment is based on the investigation of the $\mathrm{Kr}$ complexes and, at least for anionic Au clusters, the planar isomers are known to favor complex formation with rare gas atoms. This has been used previously to selectively measure the photoelectron spectrum of a minority $2 \mathrm{D}$ isomer of $\mathrm{Au}_{12}{ }^{-}$out of a mixture of 2D/3D isomers [99]. Nevertheless, the experimental data are in excellent agreement with the theoretical IR spectra of 10-D predicted to be most stable by the free-energy calculations.

\section{3. $\mathrm{Au} \mathbf{u}_{11}$}

The nonplanar capped-trigonal prism $\left(11-\mathrm{K}, R_{P}=0.80\right)$ of $\mathrm{Au}_{11}$ shows excellent agreement of the theoretical IR spectrum with the experimental spectrum of $\mathrm{Au}_{11} \mathrm{Kr}_{2}$ having two prominent peaks around 190 and $110 \mathrm{~cm}^{-1}$. Isomer $11-\mathrm{K}$ is also the most stable isomer compared to competing isomers based on our free-energy calculations at $100 \mathrm{~K}$. Thus, for $\mathrm{Au}_{11}$ we assign the FIR-MPD spectra to the nonplanar cappedtrigonal prism (11-K). A contribution of a minor fraction of the planar isomers $\mathrm{H}+(11-\mathrm{H} 1$ and 11-H2) could explain the additional peaks seen in the FIR-MPD spectrum of $\mathrm{Au}_{11} \mathrm{Kr}$.

\section{4. $\mathrm{Au}_{12}$}

Our free-energy calculations suggest $\mathrm{Au}_{12}$ most likely adopts a planar di-capped elongated-hexagon structure (12-O) at $100 \mathrm{~K}$, whereas our theoretical IR spectra and experimental FIR-MPD spectra for $\mathrm{Au}_{12} \mathrm{Kr}$ and $\mathrm{Au}_{12} \mathrm{Kr}_{2}$ show good agreement for a nonplanar cup $\left(\mathrm{C}_{2 v}\right)$ isomer (Fig. 11, 12-P). Although 12-V has a similar IR spectrum to 12-P, it is much less stable. This structural assignment is further supported by the theoretical harmonic IR spectra of 12-P, which shows peaks around 135,175 , and $185 \mathrm{~cm}^{-1}$ with similar intensity, whereas $12-\mathrm{V}$ does not (Fig. S12 in [91]). Isomer 12-P is 


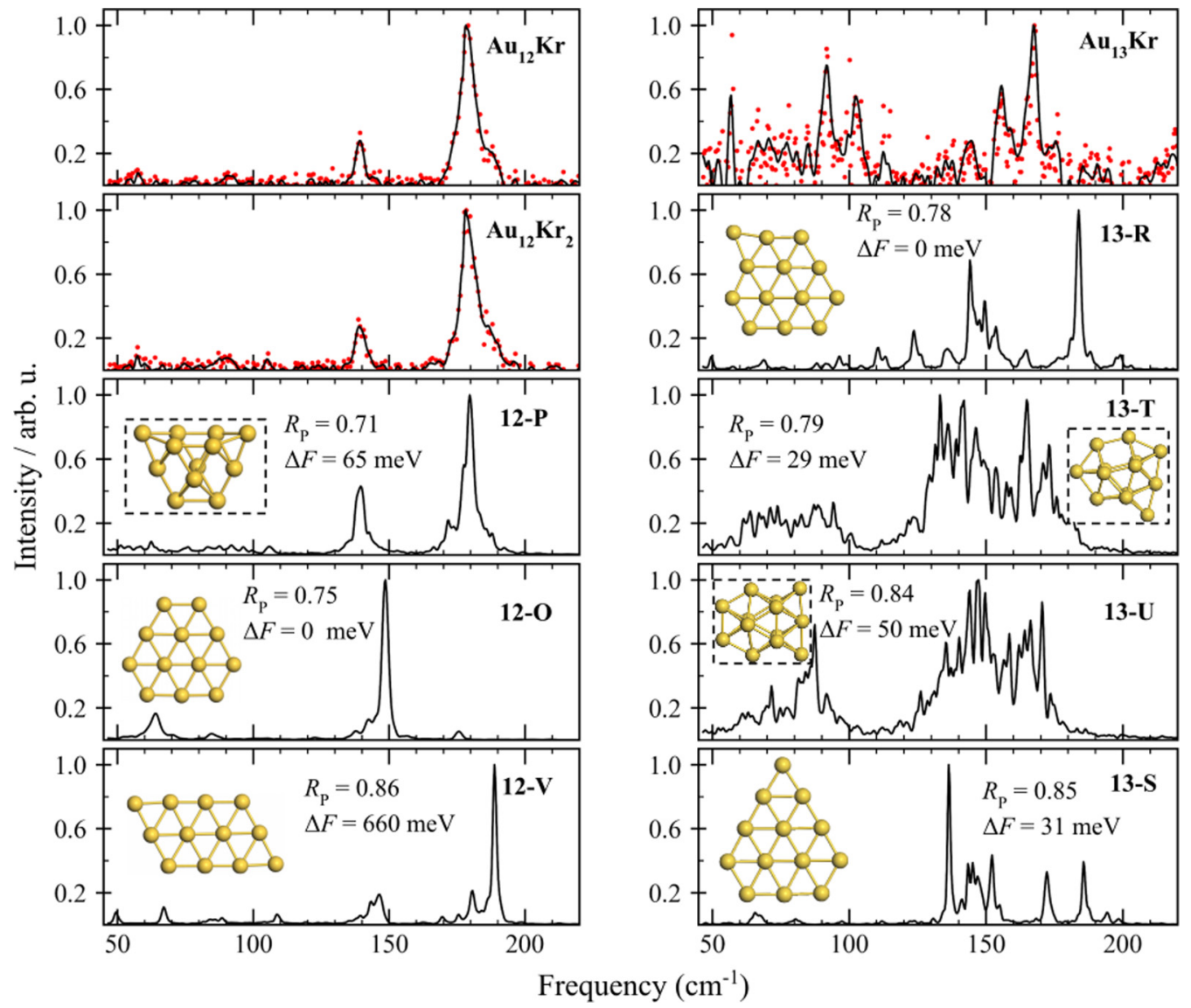

FIG. 11. Comparison of experimental FIR-MPD and theoretical anharmonic IR spectra for $\mathrm{Au}_{12}$ and $\mathrm{Au}_{13}$ at $100 \mathrm{~K}$. For each size, the upper panel(s) show the experimental spectra for the complexes with $\mathrm{Kr}$ atoms and below theoretical IR spectra are shown. Pendry $R$ factors $\left(R_{P}\right)$ comparing theoretical IR spectra with experimental results from $A u_{12} \mathrm{Kr}_{2}$ and $\mathrm{Au}_{13} \mathrm{Kr}$ are shown. Free-energy calculations ( $\Delta F$, referenced to the lowest free-energy isomer) are shown at $100 \mathrm{~K}$ for each isomer. Structures surrounded by a black-dashed box are assigned to experimental FIR-MPD spectra based on $R_{P}$ and the free-energy calculations.

found to be the second most stable isomer at $100 \mathrm{~K}$ (and the dominant one at $200 \mathrm{~K}$ and above). Interestingly, this nonplanar cup isomer 12-P has the same structure as the prevailing isomer of the $\mathrm{Au}_{12}{ }^{-}$anionic cluster $[18,45]$. One possibility is that $12-\mathrm{P}$ is kinetically trapped and cannot reach the thermodynamically accessible planar ground-state isomer $(12-\mathrm{O})$ at $100 \mathrm{~K}$ [39]. The free-energy barrier to interconvert between nonplanar 12-P and planar $12-\mathrm{O}$ is estimated to be appreciable even above $300 \mathrm{~K}$, remaining greater than $\sim 0.32 \mathrm{eV}$ at $750 \mathrm{~K}$. Another possibility is that inaccuracies in the predicted cluster isomer energy differences at $0 \mathrm{~K}$ lead to a shift in the 2D/3D crossover temperature of $\mathrm{Au}_{12}$.

\section{5. $\mathrm{Au}_{13}$}

For $\mathrm{Au}_{13}$, previous DFT studies have suggested either the planar 13-R or one of the nonplanar 13-T and 13-U isomers is the most stable $[44,57,67]$. Our DFT calculations using PBE+MBD predict $\mathrm{Au}_{13}$ to be most stable as the planar 13-R at $0 \mathrm{~K}$ (and $100 \mathrm{~K}$ ), whereas HSE06+MBD and RPA@PBE calculations predict the most stable structure is the nonplanar $13-\mathrm{T}$ at $0 \mathrm{~K}$. At $100 \mathrm{~K}$, we predict the nonplanar 13-T is $29 \mathrm{meV}$ uphill and $13-\mathrm{U}$ is $50 \mathrm{meV}$ uphill from 13-R using PBE+MBD. As evident in the IR spectra and molecular dynamics simulations, the nonplanar isomers $13-\mathrm{T}$ and $13-\mathrm{U}$ show significant dynamics even at $100 \mathrm{~K}$. Since $\mathrm{Au}_{11}$ and $\mathrm{Au}_{12}$ were determined by FIR-MPD spectroscopy to adopt nonplanar structures, it is probable that $\mathrm{Au}_{13}$ also prefers a nonplanar structure at $100 \mathrm{~K}$. Thus, we tentatively assign the $\mathrm{Au}_{13}$ structure in our FIR-MPD measurements to a mixture of the nonplanar 13-T and 13-U isomers. Although spectroscopic evidence is inconclusive on the precise structure of $\mathrm{Au}_{13}$ at $100 \mathrm{~K}$, it appears that $\mathrm{Au}_{13}$ will adopt a variety of nonplanar structures.

\section{SUMMARY}

Accurate first-principles studies, properly considering both temperature effects and long-range van der Waals interactions, are used in conjunction with experimental far-IR multiple photon dissociation (FIR-MPD) spectroscopy to identify the structures of neutral gas phase $\mathrm{Au}_{5}-\mathrm{Au}_{13}$ clusters at finite temperature. $\mathrm{Au}_{5}-\mathrm{Au}_{8}$ are predicted to adopt planar isomers at $100 \mathrm{~K}$, which agrees with prior FIR-MPD measurements and first-principles calculations. Specifically, $\mathrm{Au}_{5}$ is most stable as a trapezoid $\left(\mathrm{C}_{2 v}\right), \mathrm{Au}_{6}$ is most stable as a triangle $\left(\mathrm{D}_{3 h}\right), \mathrm{Au}_{7}$ is most stable as an edge-capped triangle $\left(\mathrm{C}_{s}\right)$, and $\mathrm{Au}_{8}$ is most stable as a fourfold edge-capped square $\left(\mathrm{D}_{4 h}\right)$. Our experimental FIR-MPD spectroscopy measurements and theoretical anharmonic IR spectra, combined with free-energy calculations, let us assign the structures of neutral $\mathrm{Au}_{9}-\mathrm{Au}_{13}$ 
clusters. Specifically, $\mathrm{Au}_{9}$ (di-capped hexagon, $\mathrm{C}_{2 v}$ ) and $\mathrm{Au}_{10}$ (elongated hexagon, $\mathrm{D}_{2 h}$ ) prefer planar isomers, whereas $\mathrm{Au}_{11}$ (capped-trigonal prism, $\mathrm{D}_{3 h}$ ), $\mathrm{Au}_{12}$ (cup, $\mathrm{C}_{2 v}$ ), and $\mathrm{Au}_{13}$ (isomers 13-T or 13-U, $C_{s}$ ) adopt nonplanar isomers at $100 \mathrm{~K}$. Thus, $\mathrm{Au}_{10}$ can be considered the largest planar structure at $100 \mathrm{~K}$. A Pendry $R$-factor analysis is used for the comparison of the theoretical and experimental spectra. Our paper shows that for IR spectra the Pendry $R$ factor is often insufficient to assign theoretical IR spectra to experimental FIR-MPD spectra. Indeed, the Pendry $R$ factor was originally developed for low-energy electron-diffraction analysis, thus it is also necessary to consider the free energy of the gold clusters to aid structural assignments. We predict that increasing temperature generally stabilizes nonplanar gold cluster structures over planar structures due to a larger entropic contribution to the free energy, apart from $\mathrm{Au}_{11}$ due to the large number of nearly energy-degenerate planar structures. Including longrange van der Waals interactions in DFT calculations has a pronounced effect on stabilizing nonplanar structures relative to planar structures and significantly improves ground-state energy predictions relative to calculations using the randomphase approximation. The impact of long-range van der Waals interactions on stabilizing nonplanar structures relative to planar structures may be general for gas phase clusters.

\section{ACKNOWLEDGMENTS}

B.R.G. acknowledges support from the Alexander von Humboldt Foundation and start-up funds from University of Michigan, Ann Arbor. This project has received funding from the European Unions Horizon 2020 research and innovation program (No. 676580: The NOMAD Laboratory - an European Center of Excellence and No. 740233: TEC1p), the Berlin Big-Data Center (BBDC, 01IS14013E), and BiGmax, the Max Planck Society's Research Network on Big-DataDriven Materials-Science. The authors thank I. Y. Zhang, J. Hermann, V. V. Gobre, D. A. Goldsmith, and A. Tkatchenko for fruitful discussions.
[1] B. Yoon, P. Koskinen, B. Huber, O. Kostko, B. von Issendorff, H. Häkkinen, M. Moseler, and U. Landman, Chem. Phys. Chem. 8, 157 (2007).

[2] E. M. Fernández and L. C. Balbás, Phys. Chem. Chem. Phys. 13, 20863 (2011).

[3] K. Baishya, J. C. Idrobo, S. Öğüt, M. Yang, K. A. Jackson, and J. Jellinek, Phys. Rev. B 83, 245402 (2011).

[4] A. B. Rahane and M. D. Deshpande, J. Phys. Chem. C 116, 2691 (2012).

[5] S. Bhattacharya, S. V. Levchenko, L. M. Ghiringhelli, and M. Scheffler, Phys. Rev. Lett. 111, 135501 (2013).

[6] N. Austin, J. K. Johnson, and G. Mpourmpakis, J. Phys. Chem. C 119, 18196 (2015).

[7] X.-N. Li, H.-M. Zhang, Z. Yuan, and S.-G. He, Nat. Commun. 7, 11404 (2016).

[8] H. Häkkinen, S. Abbet, A. Sanchez, U. Heiz, and U. Landman, Angew. Chem. Int. Ed. 42, 1297 (2003).

[9] G. Mills, M. S. Gordon, and H. Metiu, J. Chem. Phys. 118, 4198 (2003).

[10] M.-C. Daniel and D. Astruc, Chem. Rev. 104, 293 (2004).

[11] B. Yoon, H. Häkkinen, U. Landman, A. S. Wörz, J.-M. Antonietti, S. Abbet, K. Judai, and U. Heiz, Science 307, 403 (2005).

[12] P. Pyykkö, Chem. Soc. Rev. 37, 1967 (2008).

[13] H. Häkkinen, Chem. Soc. Rev. 37, 1847 (2008).

[14] H.-J. Freund, G. Meijer, M. Scheffler, R. Schlögl, and M. Wolf, Angew. Chem. Int. Ed. 50, 10064 (2011).

[15] E. C. Beret, M. M. van Wijk, and L. M. Ghiringhelli, Int. J. Quant. Chem. 114, 57 (2014).

[16] J.-X. Liu, Z. Liu, I. A. Filot, Y. Su, I. Tranca, and E. J. Hensen, Catal. Sci. Technol. 7, 75 (2017).

[17] S. M. Lang, T. M. Bernhardt, V. Chernyy, J. M. Bakker, R. N. Barnett, and U. Landman, Angew. Chem. Int. Ed. 56, 13406 (2017).

[18] X. Xing, B. Yoon, U. Landman, and J. H. Parks, Phys. Rev. B 74, 165423 (2006).

[19] S. Bulusu, X. Li, L.-S. Wang, and X. C. Zeng, Proc. Natl. Acad. Sci. USA 103, 8326 (2006).
[20] E. S. Kryachko and F. Remacle, Int. J. Quant. Chem. 107, 2922 (2007).

[21] R. M. Olson and M. S. Gordon, J. Chem. Phys. 126, 214310 (2007).

[22] P. Weis, T. Bierweiler, E. Vollmer, and M. M. Kappes, J. Chem. Phys. 117, 9293 (2002).

[23] D. Schooss, P. Weis, O. Hampe, and M. M. Kappes, Phil. Trans. R. Soc. A 368, 1211 (2010).

[24] L.-M. Wang and L.-S. Wang, Nanoscale 4, 4038 (2012).

[25] H. Grönbeck and W. Andreoni, Chem. Phys. 262, 1 (2000).

[26] J. Wang, G. Wang, and J. Zhao, Phys. Rev. B 66, 035418 (2002).

[27] E. M. Fernández, J. M. Soler, I. L. Garzón, and L. C. Balbás, Phys. Rev. B 70, 165403 (2004).

[28] K. R. S. Chandrakumar, T. K. Ghanty, and S. K. Ghosh, J. Phys. Chem. A 108, 6661 (2004).

[29] D. M. P. Mingos, Gold Clusters, Colloids and Nanoparticles I (Springer, New York, 2014), Vol. 161, p. 282.

[30] B. R. Goldsmith, M. Boley, J. Vreeken, M. Scheffler, and L. M. Ghiringhelli, New J. Phys. 19, 013031 (2017).

[31] W. Zhang, A. Sumer, J. Jellinek, and D. Cheng, Chem. Nano. Mat. 1, 482 (2015).

[32] A. F. Ramos, R. Arratia-Perez, and G. L. Malli, Phys. Rev. B 35, 3790 (1987).

[33] P. Pyykkö, Chem. Rev. 88, 563 (1988).

[34] R. Arratia-Perez, A. F. Ramos, and G. L. Malli, Phys. Rev. B 39, 3005 (1989).

[35] L. Lamare and F. Michel-Calendini, Int. J. Quant. Chem. 61, 635 (1997).

[36] H. Häkkinen, M. Moseler, and U. Landman, Phys. Rev. Lett. 89, 033401 (2002).

[37] L. Xiao and L. Wang, Chem. Phys. Lett. 392, 452 (2004).

[38] R. M. Olson, S. Varganov, M. S. Gordon, H. Metiu, S. Chretien, P. Piecuch, K. Kowalski, S. A. Kucharski, and M. Musial, J. Am. Chem. Soc. 127, 1049 (2005).

[39] P. Koskinen, H. Häkkinen, B. Huber, B. von Issendorff, and M. Moseler, Phys. Rev. Lett. 98, 015701 (2007).

[40] L. Ferrighi, B. Hammer, and G. K. H. Madsen, J. Am. Chem. Soc. 131, 10605 (2009). 
[41] S. A. Serapian, M. J. Bearpark, and F. Bresme, Nanoscale 5, 6445 (2013).

[42] D. A. Götz, R. Schäfer, and P. Schwerdtfeger, J. Comp. Chem. 34, 1975 (2013).

[43] J. A. Hansen, P. Piecuch, and B. G. Levine, J. Chem. Phys. 139, 091101 (2013).

[44] M. P. Johansson, I. Warnke, A. Le, and F. Furche, J. Phys. Chem. C 118, 29370 (2014).

[45] F. Furche, R. Ahlrichs, P. Weis, C. Jacob, S. Gilb, T. Bierweiler, and M. M. Kappes, J. Chem. Phys. 117, 6982 (2002).

[46] S. Gilb, P. Weis, F. Furche, R. Ahlrichs, and M. M. Kappes, J. Chem. Phys. 116, 4094 (2002).

[47] M. P. Johansson, A. Lechtken, D. Schooss, M. M. Kappes, and F. Furche, Phys. Rev. A 77, 053202 (2008).

[48] M. Mantina, R. Valero, and D. G. Truhlar, J. Chem. Phys. 131, 064706 (2009).

[49] J. P. K. Doye and D. J. Wales, New J. Chem. 22, 733 (1998).

[50] J. Oviedo and R. E. Palmer, J. Chem. Phys. 117, 9548 (2002).

[51] H. Häkkinen, B. Yoon, U. Landman, X. Li, H.-J. Zhai, and L.-S. Wang, J. Phys. Chem. A 107, 6168 (2003).

[52] H. Häkkinen, M. Moseler, O. Kostko, N. Morgner, M. A. Hoffmann, and B. v. Issendorff, Phys. Rev. Lett. 93, 093401 (2004).

[53] C. M. Chang and M. Y. Chou, Phys. Rev. Lett. 93, 133401 (2004).

[54] H. Zhai and A. N. Alexandrova, ACS Catal. 7, 1905 (2017).

[55] L. M. Ghiringhelli, P. Gruene, J. T. Lyon, D. M. Rayner, G. Meijer, A. Fielicke, and M. Scheffler, New J. Phys. 15, 083003 (2013).

[56] M. R. Fagiani, X. Song, P. Petkov, S. Debnath, S. Gewinner, W. Schöllkopf, T. Heine, A. Fielicke, and K. R. Asmis, Angew. Chem. Int. Ed. 56, 501 (2017).

[57] E. C. Beret, L. M. Ghiringhelli, and M. Scheffler, Farad. Discuss. 152, 153 (2011).

[58] G. Santarossa, A. Vargas, M. Iannuzzi, and A. Baiker, Phys. Rev. B 81, 174205 (2010).

[59] H. Rydberg, M. Dion, N. Jacobson, E. Schröder, P. Hyldgaard, S. I. Simak, D. C. Langreth, and B. I. Lundqvist, Phys. Rev. Lett. 91, 126402 (2003).

[60] G. A. Rance, D. H. Marsh, S. J. Bourne, T. J. Reade, and A. N. Khlobystov, ACS Nano 4, 4920 (2010).

[61] M.-T. Nguyen, C. A. Pignedoli, M. Treier, R. Fasel, and D. Passerone, Phys. Chem. Chem. Phys. 12, 992 (2010).

[62] K. Tonigold and A. Groß, J. Chem. Phys. 132, 224701 (2010).

[63] A. Ambrosetti, N. Ferri, R. A. DiStasio, and A. Tkatchenko, Science 351, 1171 (2016).

[64] V. G. Ruiz, W. Liu, E. Zojer, M. Scheffler, and A. Tkatchenko, Phys. Rev. Lett. 108, 146103 (2012).

[65] J. C. F. Rodriguez-Reyes, C. G. F. Siler, W. Liu, A. Tkatchenko, C. M. Friend, and R. J. Madix, J. Am. Chem. Soc. 136, 13333 (2014).

[66] P. Gruene, B. Butschke, J. T. Lyon, D. M. Rayner, and A. Fielicke, Z. Phys. Chem. 228, 337 (2014).

[67] A. Kinaci, B. Narayanan, F. G. Sen, M. J. Davis, S. K. Gray, S. K. R. S. Sankaranarayanan, and M. K. Y. Chan, Sci. Rep. 6, 34974 (2016).

[68] M. R. Shirts and J. D. Chodera, J. Chem. Phys. 129, 124105 (2008).
[69] V. Blum, R. Gehrke, F. Hanke, P. Havu, V. Havu, X. Ren, K. Reuter, and M. Scheffler, Comput. Phys. Commun. 180, 2175 (2009).

[70] V. Havu, V. Blum, P. Havu, and M. Scheffler, J. Comp. Phys. 228, 8367 (2009).

[71] M. Ernzerhof and G. E. Scuseria, J. Chem. Phys. 110, 5029 (1999).

[72] J. Heyd, G. E. Scuseria, and M. Ernzerhof, J. Chem. Phys. 118, 8207 (2003).

[73] T. M. Henderson, A. F. Izmaylov, G. Scalmani, and G. E. Scuseria, J. Chem. Phys. 131, 044108 (2009).

[74] A. Tkatchenko and M. Scheffler, Phys. Rev. Lett. 102, 073005 (2009).

[75] A. Tkatchenko, R. A. DiStasio, R. Car, and M. Scheffler, Phys. Rev. Lett. 108, 236402 (2012).

[76] A. Ambrosetti, A. M. Reilly, R. A. DiStasio, and A. Tkatchenko, J. Chem. Phys. 140, 18A508 (2014).

[77] A. V. Krukau, O. A. Vydrov, A. F. Izmaylov, and G. E. Scuseria, J. Chem. Phys. 125, 224106 (2006).

[78] X. Ren, P. Rinke, C. Joas, and M. Scheffler, J. Mat. Sci. 47, 7447 (2012).

[79] K. A. Peterson, D. E. Woon, and T. H. Dunning, Jr., J. Chem. Phys. 100, 7410 (1994).

[80] E. van Lenthe, A. Ehlers, and E.-J. Baerends, J. Chem. Phys. 110, 8943 (1999).

[81] C. M. Marian, in Reviews in Computational Chemistry (Wiley, New York, 2001), p. 99.

[82] D. J. Earl and M. W. Deem, Phys. Chem. Chem. Phys. 7, 3910 (2005).

[83] G. Bussi, D. Donadio, and M. Parrinello, J. Chem. Phys. 126, 014101 (2007).

[84] S. C. Harvey, R. K. Z. Tan, and T. E. Cheatham III, J. Comp. Chem. 19, 726 (1998).

[85] E. Braun, S. M. Moosavi, and B. Smit, J. Chem. Theory Comput. 14, 5262 (2018).

[86] C. Draxl and M. Scheffler, arXiv:1805.05039 (2018).

[87] M.-P. Gaigeot, M. Martinez, and R. Vuilleumier, Mol. Phys. 105, 2857 (2007).

[88] R. Ramírez, T. López-Ciudad, P. Kumar P, and D. Marx, J. Chem. Phys. 121, 3973 (2004).

[89] J. Pendry, J. Phys. C 13, 937 (1980).

[90] M. Rossi, Ph.D. thesis, Fritz-Haber-Institut der Max-PlanckGesellschaft and Technissche Universität, 2011.

[91] See Supplemental Material at http://link.aps.org/supplemental/ 10.1103/PhysRevMaterials.3.016002 for figures and tables.

[92] A. V. Walker, J. Chem. Phys. 122, 094310 (2005).

[93] J. J. Rehr, E. Zaremba, and W. Kohn, Phys. Rev. B 12, 2062 (1975).

[94] M. Ceriotti, G. A. Tribello, and M. Parrinello, J. Chem. Theory Comput. 9, 1521 (2013).

[95] P. Gruene, D. M. Rayner, B. Redlich, A. F. G. van der Meer, J. T. Lyon, G. Meijer, and A. Fielicke, Science 321, 674 (2008).

[96] M. Gruber, G. Heimel, L. Romaner, J.-L. Brédas, and E. Zojer, Phys. Rev. B 77, 165411 (2008).

[97] A. Fielicke, A. Kirilyuk, C. Ratsch, J. Behler, M. Scheffler, G. von Helden, and G. Meijer, Phys. Rev. Lett. 93, 023401 (2004).

[98] A. Fielicke, C. Ratsch, G. von Helden, and G. Meijer, J. Chem. Phys. 122, 091105 (2005).

[99] W. Huang and L.-S. Wang, Phys. Rev. Lett. 102, 153401 (2009). 